\title{
Environmental Mortality and Long-Run Growth
}

\author{
Ulla Lehmijoki \\ University of Helsinki and HECER
}

and

Elena Rovenskaya

International Institute for Applied Systems Analysis (IIASA)

Discussion Paper No. 252

February 2009

ISSN 1795-0562

HECER - Helsinki Center of Economic Research, P.O. Box 17 (Arkadiankatu 7), FI-00014 University of Helsinki, FINLAND, Tel +358-9-191-28780, Fax +358-9-191-28781,

E-mail info-hecer@helsinki.fi, Internet www.hecer.fi 


\title{
HECER
}

Discussion Paper No. 252

\section{Environmental Mortality and Long-Run Growth*}

\begin{abstract}
There is emerging evidence that environmental degradation increases human mortality. This paper provides a long-run consumer optimization model in which mortality is endogenous to emissions generated by production. Emissions are assumed to follow the $E K C$ path, first rising and then falling along with output. In the optimum, some deaths are accepted as an exchange for consumption. The model is estimated for the European outdoor air pollution data, showing that Europe has reached the downward sloping segment of the EKC. Economic growth will thus decrease rather than increase air pollution and the associated deaths in most countries in Europe.
\end{abstract}

JEL Classification: Q01, Q53, Q54, J11

Keywords: optimal growth, pollution, environmental mortality, Europe

Ulla Lehmijoki

HECER

University of Helsinki

P.O. Box 17

FI-00014 University of Helsinki

FINLAND

e-mail: ulla.lehmijoki@helsinki.fi
Elena Rovenskaya

IIASA

International Institute for Applied

Systems Analysis

Schlossplatz 1, A-2361 Laxenburg AUSTRIA

e-mail:erovem@mail.ru

* The authors are grateful to Heikki Ruskeepää for his helpful comments on Mathematica programming. 


\section{Introduction}

As several branches of science provide new findings concerning air pollution, climate change, salination of ground water, and pollution of the ocean, there is emerging evidence that environmental degradation harms human health, and the fear that economic growth increases this degradation is common.

This paper offers a long-run economic growth model in which population growth, through mortality, is endogenous to environmental degradation. In this framework, health - and even life - is one of the competing goals of utility-maximizing agents as there is a trade-off between consumption and mortality. Nevertheless, the association between economic degradation and economic growth is not linear. On the contrary, according to the Environmental Kuznets Curve hypothesis, $E K C$, pollution first increases but then decreases along with output (Selden and Song 1994, Arrow et al. 1995, Grossman and Krueger 1995).

Our model is derived from several building blocks and several simplifications are needed to keep it tractable. Mortality increases as a response to emissions generated as by-products of production. Since emissions mostly have their roots in energy combustion, it has been argued that the capitalintensive sectors are "dirtier" than the labor-intensive ones (Cole and Elliott 2003). We take this argument to the extreme by assuming that there is only one capital-intensive sector, where capital is the sole factor of production. Hence, environmental mortality causes no negative effect on production and is important only because of welfare losses, which are modeled through the Benthamian utility function depending on per capita consumption and the population size. We assume that pollution is a public good consumed by all in equal amounts, so that only its overall extent is important. The $E K C$ hypothesis is, therefore, considered in total rather than in per capita terms. Since our emphasis is on the basic trade-off between output and deaths, many important elements such as emission-limiting policies and health-promoting 
medical efforts are left out of the model (Stokey 1998).

There seems to be a consensus that of the several environmental hazards, outdoor air pollution currently causes the greatest risk to human health. Air pollution mortality was first reported in the Meuse Valley, Belgium (1930) and London (1953), where smog took the lives of 60 and 4000 people respectively (Logan 1953, Nemery et al. 2001). Air pollution raises mortality mainly through increases in respiratory and cardiovascular diseases and lung cancer, but an increase in skin cancer has also been reported (Samet et al. 2000, Brunekreef and Holgate 2002). All age groups are affected, but the unborn, young children, and the elderly are the most vulnerable.

$C A F E$, the Clean Air for Europe program and WHO, the World Health Organization, in their recent research, have provided the first-ever estimates of environmental mortality in Europe, showing that nowadays there are more than 300000 premature deaths annually (WHO 2004). Hence, the relevant question arises of what the effect of economic growth on this number will be in the future.

To answer this question, we estimate the parameters of the model to accommodate it to the European economic and air pollution data. The critical question here is whether the European countries have already passed the $E K C$ peak. Since the derived results suggest that this is the case, our conclusion is that economic growth will decrease rather than increase the number of environmental deaths. The decrease is most rapid in countries with the lowest population growth and the highest economic growth. Nevertheless, in a sub-sample of fourteen European countries for which the complete analysis is possible, the total number of air pollution deaths from 2000 to 2020 accounts for more than four million, showing that even though Europe is one of the cleanest places in the world, its environmental deaths are numerous enough to be taken seriously.

The paper is organized as follows: Section 2 introduces the model and Section 3 works out its solution. Section 4 provides its application to the 
European air pollution deaths with country-specific results and Section 5 discusses the findings and closes the paper. The appendixes contain technical details and the data.

\section{The model}

Consider an economy in which capital $K$ is the only input, implying that the role of labor $L$ as input is negligible. The production function takes the Cobb-Douglas formula

$$
Y=A K^{\alpha}
$$

where $A>0$ is the technology level and $0<\alpha<1$ is the elasticity of the output with respect to capital.

All emissions $E$ are generated as by-products of production. Hence, for all $Y \geq 0$ emissions become

$$
E=g(Y)=g\left(A K^{\alpha}\right)
$$

We assume that the Environmental Kuznets Curve hypothesis EKC holds, i.e., emissions first rise and then fall along with the output. Therefore, the emission function $g(\cdot)$ assumes

$$
g^{\prime}(Y)>0 \text { for } Y<\mu, \quad g^{\prime}(Y)=0 \text { for } Y=\mu, \quad g^{\prime}(Y)<0 \text { for } Y>\mu,
$$

and

$$
\lim _{Y \rightarrow 0} g^{\prime}(Y)<\infty, \quad \lim _{Y \rightarrow \infty} g^{\prime}(Y)=0,
$$

where $\mu$ refers to the $E K C$ peak. ${ }^{1}$ The limit conditions (3) imply that emissions step in slowly and ultimately level off. The emission function also satisfies

$$
g(0)=0, \quad \lim _{Y \rightarrow \infty} g(Y) \geq 0
$$

\footnotetext{
${ }^{1} E K C$ arises when the emission-decreasing technology and composition effects dominate the emission-increasing scale effect, consisting of an increase in per capita incomes and population size. Since we assume that emissions are commonly "consumed" by all, i.e., emissions are a public good, only their total amount is important here. Air pollution is a good example of this.
} 
Emissions cause unwanted health consequences ranging from eye irritation to severe illness and death. We concentrate on deaths. If frequent, environmental deaths manifest themselves as a decrease in population growth. Hence, we assume that the population growth rate $\dot{L} / L=n$ consists of two components, an autonomous component and a component describing the environmental deaths. Concentrating on the latter, we assume that the autonomous component $\nu$ is constant. Hence, for all $E \geq 0$, the population growth rate $n=n(E)$ satisfies

$$
n(0)=\nu>0, \quad n^{\prime}(E)<0
$$

indicating that population growth is positive for zero emissions but decreases as emissions increase. Note that since the production function (1) is highly stylized, environmental mortality induces no feedback on the output. This is justified if most environmental victims are children and elderly adults as is the case of the air pollution deaths analyzed here.

Noting (4) and normalizing the initial population to unity, the population size at time $t$ becomes

$$
L(t)=\exp \int_{0}^{t} n[E(\tau)] d \tau
$$

Since the output can be either consumed or saved, the capital stock accumulates according to

$$
\dot{K}=A K^{\alpha}-C-\delta K, \quad K(0)=K_{0},
$$

where $\delta>0$ refers to depreciation and $K_{0}$ to a positive initial value of the capital stock.

Consider a benevolent central planner facing the Benthamian societal utility $u(C / L) \cdot L$, which depends on individual utility from per capita consumption $C / L$ and the number of people $L$. Environmental deaths thus cause disutility to the planner. To keep the model simple, environmental amenities 
are not included. Let the individual utility function adopt the CIES formula

$$
u(C / L)=\frac{(C / L)^{1-\theta}}{1-\theta}, \quad(\theta \neq 1) .^{2}
$$

The central planner thus chooses consumption $C(\cdot)$ to maximize the utility index

$$
\begin{aligned}
U & =\int_{0}^{\infty} u[C(t) / L(t)] L(t) e^{-\rho t} d t \\
& =\int_{0}^{\infty} \frac{C(t)^{1-\theta}}{1-\theta} e^{-\int_{0}^{t}\{\rho-\theta n[E(\tau)]\} d \tau} d t
\end{aligned}
$$

subject to (2), (3), (4), (6). To keep (7) bounded, let

$$
\rho-\theta \nu>0 .^{3}
$$

\section{Optimal Consumption and Investment}

The fact that the discount factor $\Delta(t)=\int_{0}^{t}\{\rho-\theta n[E(\tau)]\} d \tau$ in $(7)$ is not constant provides difficulties for the analysis. To eliminate them, we apply the virtual time technique suggested by Uzawa (1968). Given (8), the factor $\Delta(t)$ has the following properties:

(i) $\Delta(0)=0$,

(ii) $\Delta(\infty)=\infty$,

(iii) $\Delta(t)$ is monotonically increasing with $\dot{\Delta}(t)=\rho-n[E(t)]>0$.

Since $\Delta(t)$ satisfies the regularity conditions suggested by Uzawa (1968) and can be used as an alternative independent time variable, we set $C=C(\Delta)$, $K=K(\Delta), E=E(\Delta)$. Furthermore,

$$
d t=\frac{d \Delta(t)}{\rho-\theta n[E(t)]}=\frac{d \Delta}{\rho-\theta n[E(\Delta)]} .
$$

\footnotetext{
${ }^{2}$ The alternative specification, $u(C / L)=\frac{(C / L)^{1-\theta}}{1-\theta}-1$, has the convenient property $\lim _{\theta \rightarrow 1} u(C / L)=\ln C / L$, but this fails in the shorter expression above. Hence the requirement $\theta \rightarrow 1$
$\theta \neq 1$ . Both formulas lead to the same results.

${ }^{3}$ Note that due to (4), assumption (8) is sufficient for the positiveness of $\rho-\theta \nu(E)$ for all $E \geq 0$.
} 
Applying (9) to (2) - (7) turns the problem into

$$
\begin{aligned}
\operatorname{maximize}_{C(\cdot)} \quad U & =\int_{0}^{\infty} \frac{C^{1-\theta}}{(1-\theta)(\rho-\theta n(E))} e^{-\Delta} d \Delta, \\
\text { subject to: } \stackrel{\circ}{K} & =\frac{d K}{d \Delta}=\frac{A K^{\alpha}-C-\delta K}{\rho-\theta n(E)}, \quad K(0)=K_{0}, \\
E & =g\left(A K^{\alpha}\right) .
\end{aligned}
$$

Problem (10) is equivalent to (2) - (7) and can be solved in virtual time by the Pontryagin maximum principle for optimal control problems on the infinite time horizon (as in Aseev and Kryazhimskiy 2007). Letting $\lambda$ be the adjoint variable, the Hamiltonian and necessary conditions become:

$$
\begin{aligned}
H(K, C, \lambda) & =\frac{1}{\rho-\theta n(E)}\left\{\frac{C^{1-\theta}}{1-\theta}+\lambda\left[A K^{\alpha}-C-\delta K\right]\right\}, \\
\frac{\partial H}{\partial C} & =0 \Longleftrightarrow C^{-\theta}=\lambda, \\
\lambda & =\frac{d \lambda}{d \Delta}=-\frac{\partial H}{\partial K}+\lambda .
\end{aligned}
$$

Since

$$
\dot{\lambda}=(d \lambda(\Delta) / d \Delta) \cdot(d \Delta / d t)=\dot{\lambda}(\rho-\theta n(E)),
$$

multiplying (13) by $(\rho-\theta n(E))$ transforms it back to the natural time and, after some algebra, the equation for the adjoint variable becomes ${ }^{4}$

$$
\dot{\lambda} / \lambda=-\left\{\frac{\theta n^{\prime} g^{\prime} \alpha A K^{\alpha-1}}{\lambda} H+\alpha A K^{\alpha-1}-\delta-\rho+\theta n\right\} .
$$

To eliminate $\lambda$, take the time derivative of (12) and insert into (14) to get

$$
\begin{aligned}
\dot{C} & =\frac{1}{\theta}\left\{\frac{\theta n^{\prime} g^{\prime} \alpha A K^{\alpha-1}}{\lambda} H+\alpha A K^{\alpha-1}-\delta-\rho+\theta n\right\} \\
& =\frac{1}{\theta}\left\{\frac{\theta n^{\prime} g^{\prime} \alpha A K^{\alpha-1}}{\rho-\theta n}\left(\frac{\theta C}{1-\theta}+A K^{\alpha}-\delta K\right)+\alpha A K^{\alpha-1}-\delta-\rho+\theta n\right\} .
\end{aligned}
$$

Equations (6) and (15) supply the solution of the model. The phase lines are:

$\frac{\dot{C}}{C}=0 \Leftrightarrow C=\frac{\theta-1}{\theta}\left\{A K^{\alpha}-\delta K+\frac{\rho-\theta n}{\theta n^{\prime} g^{\prime} \alpha A K^{\alpha-1}}\left[\alpha A K^{\alpha-1}-\delta-\rho+\theta n\right]\right\}$, $\dot{K}=0 \Leftrightarrow C=A K^{\alpha}-\delta K$.

\footnotetext{
${ }^{4}$ To make the formulas shorter, we leave out the arguments of the functions if possible.
} 
The phase line $\dot{K}=0$ with slope $\alpha A K^{\alpha}-\delta$ is strictly concave, reaches its maximum at $\bar{K}=(\delta / \alpha A)^{1 /(\alpha-1)}$, and hits the horizontal axis at the origin and $\tilde{K}=(\delta / A)^{1 /(\alpha-1)}$, as is illustrated in Figure 2. All steady states, if they exist, are allocated on the upward sloping segment of $\dot{K}=0$. For the $E K C$ to be of economic relevance, it should thus peak at lower values of $K$. Hence, we assume $\mu<\bar{K}$.

The phase line $\dot{C} / C=0$ can adopt several shapes depending upon the values of the parameter $\theta$. Since Hall (1988) has argued that empirical elasticities tend to be large, we assume $\theta>1$, implying that the fraction $(\theta-1) / \theta$ is positive. ${ }^{5}$ The fact that $g^{\prime}$ changes its sign at the $E K C$ peak implies that the line $\dot{C} / C=0$ has a point of discontinuity at $K=\mu$. Since

$$
0 \leq A K^{\alpha}-\delta K<\infty
$$

for all $K<\tilde{K}$, the limit behavior of $\dot{C}=0$ depends on its rightmost expression

$$
\frac{\rho-\theta n}{\theta n^{\prime} g^{\prime} \alpha A K^{\alpha-1}}\left[\alpha A K^{\alpha-1}-\delta-\rho-\theta n\right] .
$$

Thus, noting (2), (3), (4) and (8), it holds that

$$
\begin{aligned}
\lim _{K \rightarrow 0} C & =\frac{\theta-1}{\theta} \frac{\rho-\theta n(0)}{\theta n^{\prime}(0) g^{\prime}(0)}<0, \\
\lim _{K \uparrow \mu} C & =-\infty \\
\lim _{K \downarrow \mu} C & =+\infty \\
\lim _{K \rightarrow \tilde{K}} C & =\frac{\theta-1}{\theta} \frac{\rho-\theta n\left[g\left(A \tilde{K}^{\alpha}\right)\right]}{\theta n^{\prime}\left[g\left(A \tilde{K}^{\alpha}\right)\right] g^{\prime}\left(A \tilde{K}^{\alpha}\right) \alpha A \tilde{K}^{\alpha-1}}\left[\alpha A \tilde{K}^{\alpha-1}-\delta-\rho+\theta n\left[g\left(A \tilde{K}^{\alpha}\right)\right]\right] \\
& <0 .
\end{aligned}
$$

Hence, $\dot{C} / C=0$ adopts an inverted-U shaped graph for $K<\mu$ but swings from $\infty$ to negative values when $K>\mu$. Inequality (16) affects the shape of $\dot{C} / C=0$ in the vicinity of the $K$-axis. Figure 2 illustrates this.

Figure 1 shows that two cases are possible. The inverted-U part of the phase line $\dot{C} / C=0$ can lie low enough to avoid the intersection with $\dot{K}=0$.

\footnotetext{
${ }^{5}$ The case where $\theta<1$ can, however, be considered analogously.
} 


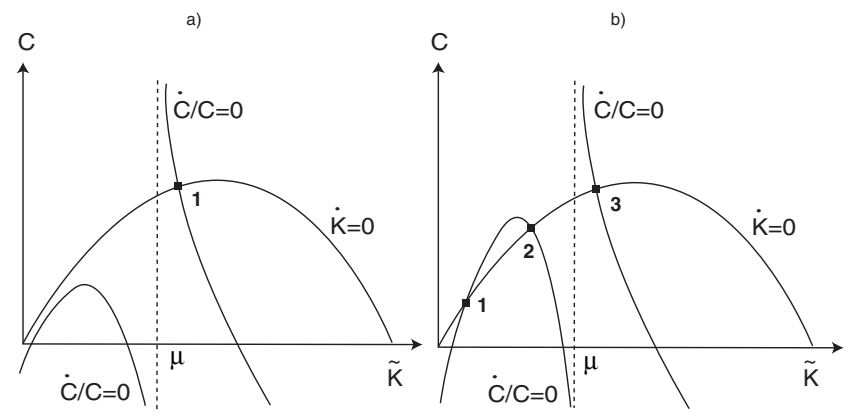

Figure 1: The phase lines.

In this case, the number of interior steady states is one. ${ }^{6}$ Alternatively, the inverted-U part of $\dot{C}=0$ may lie so high that it intersects $\dot{K}=0$. In this case, the number of interior steady states is three. The former is given in panel $a$ and the latter in panel $b$ in Figure 1. A standard local stability analysis in Appendix A shows that steady states 1 and 3 are saddles with stable branches running from southwest and northeast while steady state 2 is an unstable focus or node. These results are summarized as follows:

Proposition. The problem $(2)-(7)$ has at least one steady state $\left(K^{*}, C^{*}\right)$, in which $K^{*}>0, C^{*}>0$ and $K^{*}<\tilde{K}=(\delta / A)^{1 /(\alpha-1)}$.

One can comprehend the model by comparing it with the standard model of optimal growth in which the central planner only faces the trade-off between current consumption and future consumption streams, whereas in the present model she also faces the trade-off between future consumption streams and environmental deaths. Since high future consumption calls for investment and production, inducing higher emissions and more numerous deaths, the planner evaluates current consumption against the future consumption net of induced deaths. In the optimum, she accepts some deaths as an exchange for the sufficient consumption.

\footnotetext{
${ }^{6}$ The non-generic case in which $\dot{C}=0$ is a tangent to $\dot{K}=0$ is not analyzed. Because of the discontinuity and non-concavities in the phase lines, additional intersections can not be excluded a priori. The emission function may also exhibit several peaks, giving rise to several points of discontinuity without violating the basic structure of the model.
} 


\section{Air Pollution Mortality in Europe}

$C A F E$, the Clean Air for Europe program and $W H O$ have recently provided summary estimates of mortality caused by short-term exposure in Europe by collecting 629 time-series and 160 individual or panel studies that regress daily mortality against daily changes in outdoor air pollution (WHO 2004). These summary estimates show that there is a significant response in mortality to particulate matter $(P M)$ and ozone. ${ }^{7}$ Pope et al. (2002) have analyzed the effects of long-term $P M$ exposures in the United States in a study in which questionnaires monitored individuals from 1982 onwards, making control for other risk sources possible. Their estimates were applied to the European data to derive the effects of long-term exposure; the short-term and long-term exposures together induced more than 300000 premature deaths in 2000 in Europe (WHO 2004). ${ }^{8}$

Although the available mortality numbers refer to several pollutants, most deaths are caused by particulate matter (WHO 2004). Furthermore, as particulates are closely associated with other pollutants, they can be used as an indicator of outdoor air pollution (Cohen et al. 2004). Thus, we concentrate on particulate matter here. The data comes from Amann et al. (2007), who report the $P M_{2.5}$ emissions for 25 European countries $\left(E U_{25}\right)$ for the year 2000. Appendix B gives the list of countries together with mortality and emission data.

\footnotetext{
${ }^{7}$ Particulate matter, $P M$, consists of solid airborne particles of varying size, chemical composition, mainly generated by energy combustion (mobile or fixed site), often also from long-distance sources. Particulate matter is further classified according to its maximum diameter size, the main groups being $P M_{2.5}$ and $P M_{10}$ with maximal diameters of 2.5 and $10 \mu \mathrm{m}$ respectively.

${ }^{8}$ For methodological issues in epidemiological studies, see Chay et al. (2003). For studies on infant mortality, see Chay and Greenstone (2003) and Currie and Neidell (2005). For techniques for deriving country-level mortality estimates, see Ostro (2004).
} 


\subsection{Generating the Missing Emission Data}

The theoretical model claims that the emission function $E=g(Y)=g(G D P)$ should adopt an $E K C$ consistent path, i.e., emissions should first rise and then fall as a function of output $Y$ measured as the real gross domestic product $G D P$. To estimate such a function, several observations of emissions and $G D P$ are necessary but, unfortunately, the $P M_{2.5}$ data above is available only for the year 2000. We thus estimate the emission-output association from a cross-section of countries in 2000 and then generalize this association to time series in individual countries.

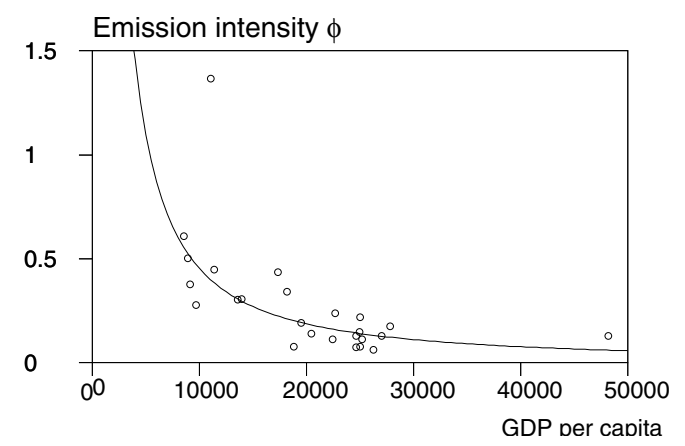

Figure 2: Emission intensity and per capita GDP in $E U_{25}$.

For the cross-section, we calculate the emission intensity of output $\phi=$ $E / G D P$ to regress it against the value of the real per capita domestic product $G D P p c$ in each $E U_{25}$ country. Figure 2 shows that $\phi$ decreases as a function of $G D P p c$, implying that richer countries apply cleaner production methods. A suitable formula for the association seems to be $\phi=\gamma \cdot G D P p c^{\vartheta}$. Hence, by taking logs, we fit

$$
\ln \phi_{i}=\ln \gamma+\vartheta \cdot \ln G D P p c_{i}+\varepsilon_{i}
$$

by $O L S$, to derive the estimates $\gamma=56298.77$ and $\vartheta=-1.27$. Model (17) explains $55 \%$ of the cross-country variation in $\phi$.

To derive the country-specific time series from (17), we calculate $\phi_{i, t}$ by applying $G D P p c_{i, t}$ for each available year $t$ for each country $i$. Since $\phi=$ 
$E / G D P$ we get

$$
E=\phi \cdot G D P=\phi \cdot G D P p c \cdot L=56298.77 \cdot G D P p c^{-0.27} \cdot L
$$

showing that the elasticity of emissions in terms of GDPpc (population) is negative (unity). Hence, we get

$$
E_{i, t}=l_{i} \cdot 56298.77 \cdot G D P p c_{i, t}^{-0.27} \cdot L_{i, t}
$$

where the multiplicative country-specific fixed factor $l_{i}=\varepsilon_{i} / \phi_{i, 2000}$ is derived from the residual error in (17).

For most countries in $E U_{25}$, the GDPpc and population data are given from 1950 onwards in Heston et al. (2006), but for the former Soviet satellites, the GDPpc data start from 1970 (or from 1993 in some cases) and are markedly volatile. Furthermore, in the parameter estimates below, the parameter constraints $0<\alpha<1$ and $\rho-\theta \nu>0$ are not met by these countries (Hungary being an exception), while of the old EU members, only Luxemburg violates them. Hence, we perform the complete analysis for 14 countries $\left(E U_{14}\right)$, presenting $67 \%$ of the population in $E U_{25}$. The generated $P M_{2.5}$ emission time series, indexed to $1950=100$, are shown in Figure 3 showing decreasing trends, interrupted by short booms in some cases. Only in The Netherlands, emissions are increasing, showing a very recent peak.

a)

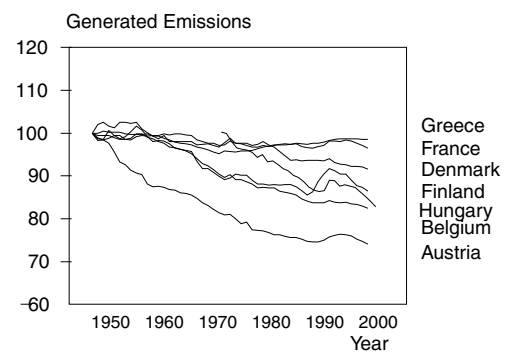

b)

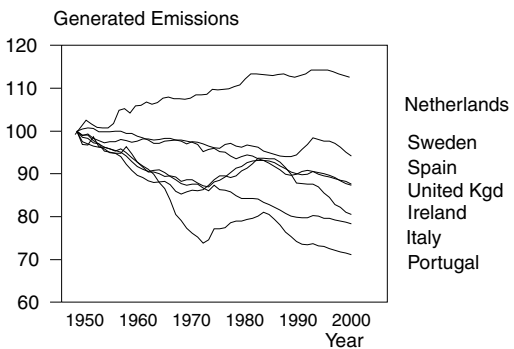

Figure 3: The generated emissions data for $E U_{14} ; 1950=100$. 


\subsection{Estimating the Country-Specific Parameters}

To apply cross-country results to a single-country model, some intermediate steps are usually needed and this holds in our case as well. Given that the cross-sectional comparison of emission intensities is meaningful only in terms of per capita GDP, the expression for emissions in (18) depends on $G D P p c$ and $L$. For mathematical reasons, however, the model above takes emissions directly as a function of GDP, claiming that the function $E=$ $g(Y)=g(G D P)$ satisfies (2) and (3), i.e., it should be consistent with the $E K C$ path and emissions should increase slowly and ultimately level off. To estimate such an emission function, we take the generated time series for emissions as plain data, and regress them against the time series for GDP in each country. A simple, feasible formula is

$$
E(t)=\eta \cdot \operatorname{Exp}\left\{-\left(\frac{G D P(t)-\mu}{\sigma}\right)^{2}\right\},
$$

where $\mu$ and $\eta$ refer to the GDP and emissions at the EKC peak, respectively. Given the downward-sloping trend in most countries (Fig. 3), the actual peak of the $E K C$ remains unknown. In these cases, the peak is allocated to the earliest available year (1950, 1970 for Hungary), but the observed peak is applied if available. For The Netherlands, the peak is allocated at 1996. By calculating $x_{t}=\left(G D P_{t}-\mu\right)^{2}$ for $t=1950-2000$ (1970 - 2000 for Hungary) for each country and taking logs, we estimate

$$
\ln E_{t}=\ln \eta+s x_{t}+\varepsilon_{t},
$$

for each country separately to derive the country-specific estimates for $\eta$ and $\sigma=-s^{0.5}$. All countries exhibit statistically highly significant values for $\sigma$. The estimates and the values for $R^{2}$ from (19) are reported in Table 1.

To evaluate $A$ and $\alpha$ in the production function $Y=G D P=A K^{\alpha}$ in (1), we first apply the standard perpetual inventory method (Caselli 2004) to generate the capital stocks from 1950 to 2000 (from 1970 for Hungary) by 
accumulating investments (data from Heston et al. 2006). We assume that the depreciation rate $\delta=0.05$ is the same in all countries. By taking logs, we then fit

$$
\ln G D P_{t}=\ln A+\alpha \cdot \ln K_{t}+\varepsilon_{t}
$$

for each country separately to derive the country-specific estimates for $\alpha$ and $A$ (Table 1).

\begin{tabular}{|l|rrr|rrr|rr|r|rr|}
\hline \hline Country & $\eta$ & $\sigma$ & $R^{2}$ & $\mathrm{~A}$ & $\alpha$ & $R^{2}$ & $\nu$ & $\beta$ & $\rho$ & $\theta$ & $R^{2}$ \\
\hline Austria & 34.77 & 364.35 & 0.82 & 1.32 & 0.78 & 1.00 & 0.0031 & $2.41 \mathrm{E}-05$ & 0.055 & 6.04 & 0.93 \\
Belgium & 39.84 & 425.03 & 0.92 & 0.70 & 0.89 & 1.00 & 0.0035 & $3.81 \mathrm{E}-05$ & 0.073 & 7.63 & 0.86 \\
Denmark & 29.37 & 313.99 & 0.92 & 1.21 & 0.78 & 0.99 & 0.0045 & $2.36 \mathrm{E}-05$ & 0.080 & 7.64 & 0.88 \\
Finland & 32.57 & 222.69 & 0.80 & 0.76 & 0.82 & 1.00 & 0.0051 & $8.70 \mathrm{E}-06$ & 0.046 & 4.62 & 0.79 \\
France & 347.59 & 7854.77 & 0.65 & 2.26 & 0.77 & 1.00 & 0.0070 & $2.17 \mathrm{E}-06$ & 0.059 & 6.80 & 0.95 \\
Greece & 48.70 & 546.28 & 0.29 & 1.60 & 0.73 & 1.00 & 0.0074 & $1.39 \mathrm{E}-05$ & 0.079 & 4.89 & 0.80 \\
Hungary & 80.88 & 130.62 & 0.95 & 2.86 & 0.64 & 0.96 & 0.0018 & $2.41 \mathrm{E}-05$ & 0.040 & 8.16 & 0.48 \\
Ireland & 16.62 & 209.32 & 0.63 & 1.10 & 0.80 & 0.98 & 0.0049 & $2.18 \mathrm{E}-05$ & 0.048 & 8.54 & 0.77 \\
Italy & 184.97 & 2370.85 & 0.93 & 0.89 & 0.88 & 1.00 & 0.0041 & $5.85 \mathrm{E}-06$ & 0.075 & 5.41 & 0.92 \\
Netherlds & 27.92 & 810.20 & 0.96 & 0.43 & 0.96 & 1.00 & 0.0090 & $3.66 \mathrm{E}-05$ & 0.066 & 6.86 & 0.88 \\
Portugal & 98.24 & 291.53 & 0.77 & 1.70 & 0.76 & 1.00 & 0.0039 & $6.42 \mathrm{E}-06$ & 0.063 & 8.30 & 0.80 \\
Spain & 166.08 & 2832.71 & 0.50 & 2.74 & 0.73 & 1.00 & 0.0075 & $3.25 \mathrm{E}-06$ & 0.051 & 6.20 & 0.81 \\
Sweden & 27.54 & 717.85 & 0.71 & 0.97 & 0.83 & 1.00 & 0.0047 & $1.46 \mathrm{E}-05$ & 0.069 & 7.92 & 0.83 \\
United Kd & 126.22 & 2773.21 & 0.95 & 8.90 & 0.61 & 0.99 & 0.0033 & $6.16 \mathrm{E}-06$ & 0.045 & 13.37 & 0.80 \\
\hline \hline
\end{tabular}

Table 1: The parameters.

Consider next the demographic function $n=n(E)$ in (4). Since most epidemiological studies indicate that the association between mortality and pollution is linear (Samet et al. 2000, Brunekreef and Holgate 2002, Pope et al. 2002), we assume $n=n(E)=\nu-\beta E$. For the autonomous population growth $\nu$, we adopt the average annual population growth rate from 1950 to 2000 in each country (Table 1). Air pollution naturally has some effect on this number since urban pollution used to be more severe than currently, but given the long time-span and large population included, this effect seems negligible. Since $\beta E$ is the death rate from air pollution, we calculate

$$
\beta=\frac{\text { air pollution deaths }}{\text { population }} \cdot E
$$


for each country separately to derive the country-specific estimates for $\beta$ (Table 1).

The average real interest rate in each country taken from the post-oil crisis period (1983-2000) has been chosen as the proxy for the time preference factor $\rho$ (data from World Bank 2008). To estimate $\theta$, consider equation (15). Unfortunately, (15) cannot be solved for $\theta$, but we simplify it by setting $n^{\prime}=0$ for $t=1950-2000$ for all countries to get $\dot{C} / C=(1 / \theta)\left(\alpha A K^{\alpha-1}-\delta-\rho\right)+$ $n .{ }^{9}$ Hence, given the country-specific values for $A, \alpha, \rho$, and $\delta=0.05$ we estimate $1 / \theta$ from

$$
\frac{\dot{C}_{t}}{C_{t}}-n_{t}=\frac{1}{\theta}\left(\alpha A K_{t}^{\alpha-1}-\delta-\rho\right)+\varepsilon_{t}
$$

for each country separately to derive the country-specific estimates for $\theta$ (Table 1).

\subsection{Results}

Given the estimated parameters, one can calculate the solution of the model and build projections of emissions and air pollution deaths for each country in $E U_{14} \cdot{ }^{10}$ The time horizon chosen extends from 2000 to 2020. The main results are shown in Table 2.

The first column in Table 2 shows calculated $P M_{2.5}$ emissions in 2020, indicating that the total annual emissions in $E U_{14}$ will decrease from 1097.34 kilotons in 2000 (Appendix B) to 899.66 kilotons in 2020. To compare the

\footnotetext{
${ }^{9}$ We evaluated the average maximal error in $E U_{14}$ from omitting the element $\frac{1}{\theta}\left\{\frac{\theta n^{\prime} g^{\prime} \alpha A K^{\alpha-1}}{\rho-\theta n}\left(\frac{\theta C}{1-\theta}+A K^{\alpha}-\delta K\right)\right\}$ in (16) by noting that this term is an increasing function of $\theta$ and that the calibrated value for $\theta$ never exceeds 15 . Thus, by assuming $\theta=15$ and calculating the omitted element for all countries we see that the average maximal (relative) error in the right hand side of (21) is $0.48 \%$.

${ }^{10}$ All results are derived by the time-elimination method, in which the stable saddle path is calculated from the steady state $\left(K^{*}, C^{*}\right)$ backwards to the origin (Mulligan and Sala-iMartin 1991). Mathematica 5.2 programs are available from the authors on request. Only a single steady state arises in every $E U_{14}$ country. The accuracy of the model, measured by its ability to meet the actual data point of air pollution deaths in 2000 is satisfactory, the average error in deaths being $-3.84 \%$.
} 


\begin{tabular}{|l|lllll|}
\hline \hline & $P M_{2.5}$ & Death rate & Deaths & Deaths & Growth $\%$ \\
Country & 2020 & 2020 & 2020 & $2000-2020$ & $2000-2020$ \\
\hline Austria & 16.33 & 0.00039 & 3580 & 96869 & 2.05 \\
Belgium & 20.30 & 0.00077 & 8415 & 229168 & 2.19 \\
Denmark & 22.27 & 0.00053 & 2966 & 66104 & 1.42 \\
Finland & 21.03 & 0.00018 & 1003 & 24435 & 2.10 \\
France & 321.38 & 0.00070 & 45393 & 922930 & 1.85 \\
Greece & 42.11 & 0.00059 & 7535 & 155919 & 1.61 \\
Hungary & 42.74 & 0.00103 & 7052 & 211619 & 1.58 \\
Ireland & 12.79 & 0.00028 & 1058 & 23790 & 2.12 \\
Italy & 79.51 & 0.00047 & 28971 & 863269 & 2.58 \\
Netherlands & 21.09 & 0.00077 & 13786 & 325084 & 2.58 \\
Portugal & 38.13 & 0.00024 & 3061 & 86445 & 2.02 \\
Spain & 135.52 & 0.00044 & 19433 & 413681 & 2.06 \\
Sweden & 24.20 & 0.00035 & 3280 & 69350 & 1.64 \\
United Kingdom & 102.24 & 0.00063 & 37325 & 809758 & 0.98 \\
\hline Total / Average & 899.66 & 0.00053 & 182857 & 4298419 & 1.91 \\
\hline \hline
\end{tabular}

Table 2: The results.

country-specific values, we construct an index by normalizing the values for 2000 to 100 . Figure 4 panel $a$ indicates that emissions will decrease everywhere, the largest decrease taking place in Portugal and the smallest in France. Table 2 and Appendix B also show that the average death rate from air pollution will decrease from 0.00069 to 0.00053 . The country-specific indexed death rates are illustrated in Figure 4, panel $b$.

The third column in Table 2 reports the number of air pollution deaths in 2020. A comparison with the data shows that the total in $E U_{14}$ will decrease from 220225 in 2000 to 182857 in 2020 . Expressed as an index, this decrease is from 100 to 83.03. Figure 5 shows the indexed time paths for deaths for each country, indicating that most marked gains will be achieved by Italy, Portugal, and Hungary. On the other hand, deaths will increase in France and Greece, where the decrease in emissions and deaths dates will be more than off-set by an increase in the population size, indicating more victims than before. In spite of the generally decreasing trends, the fourth column 
a)

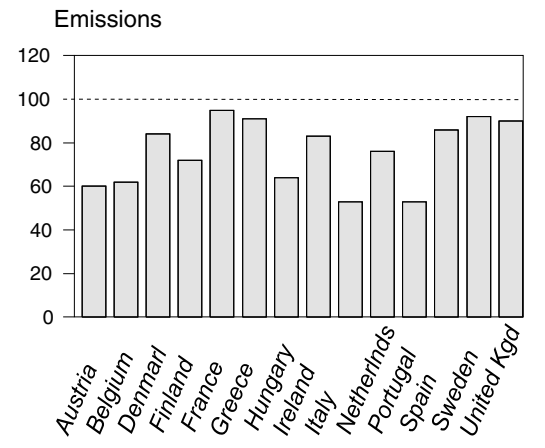

b)

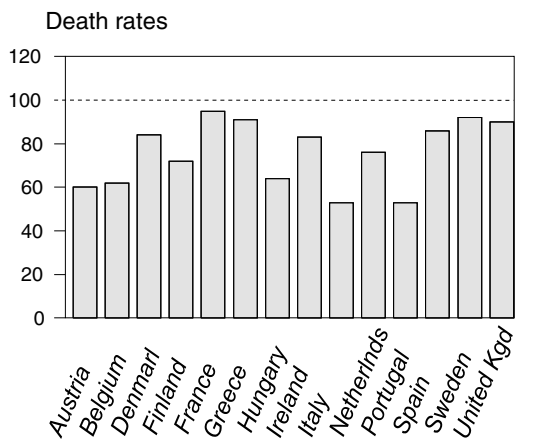

Figure 4: The index of emissions and death rates in 2020. The value for 2000 is 100 for all countries.

of Table 2 shows that the total number of air pollution deaths from 2000 to 2020 will accumulate to 4298419 persons in $E U_{14}$.

a)

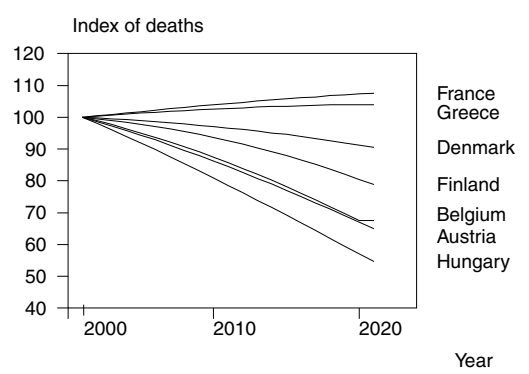

b)

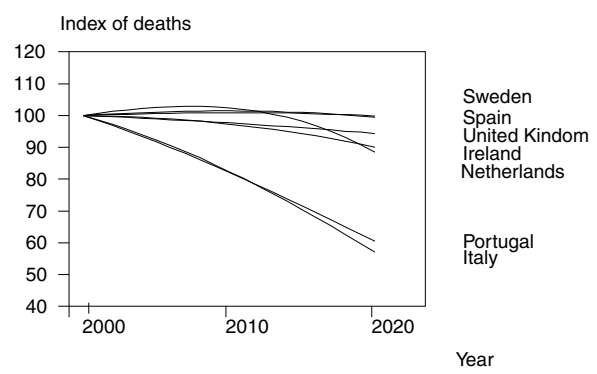

Figure 5: Trends in air pollution deaths. The value for 2000 is 100 for all countries.

In general, the importance of population growth seems to be considerable, even in an area like $E U_{14}$, where this growth is already relatively low. Figure 6 panel $a$ plots the index of air pollution deaths in 2020 against the autonomous population growth rate $(\times E-02$ of parameter $\nu)$, showing a strong positive association between them. One can see that the population growth rate is much higher in Greece (GRC), Spain (ESP) and France (FRC) than in Hungary (HUN), Italy (ITA), and Portugal (PRT), the former group 
exhibiting at most a marginal decrease in deaths, while the decrease in deaths in the latter group is large.
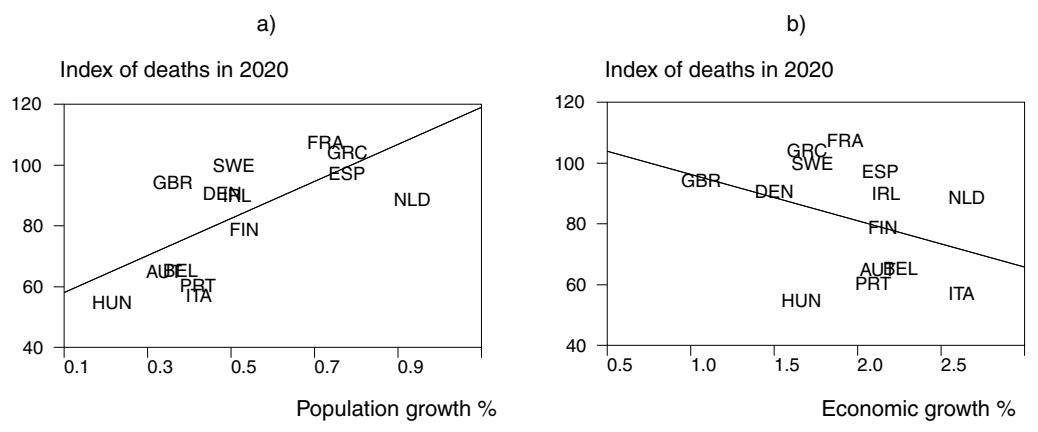

Figure 6: The index of air pollution deaths in 2020 as a function of demographic and economic growth.

On the other hand, economic growth is also important because all economies show a downward-sloping emission trend, suggesting that the fast-growers should leap ahead in their $E K C$ path. This is indeed confirmed by Figure 6 , panel $b$, which plots the death index in 2020 against the average economic growth rate from 2000 to 2020 (Table 2, last column), showing a clearly negative association. Comparison of panels $a$ and $b$ also shows several interesting cases. Italy (ITA), for example, gains a double advantage since its population growth is low and economic growth is high. On the other hand, high economic growth in The Netherlands (NLD) seems to off set the high demographic growth, although there is a delay in the decrease in deaths, caused by a slow take-off after the recent peak (Figure 5). The high death index in The United Kingdom (GBR), in turn, seems to be caused by a slow projected economic growth.

In general, the economic growth rates in $E U_{14}$ will be smaller than in the past, a result which is expected because of the decreasing productivity of capital in the model $(\alpha<1)$. Table 2 shows that from 2000 to 2020 the average economic growth rate is $1.91 \%$, while it was $2.87 \%$ from 1950 to 2000 (from 1970 to 2000 for Hungary). Hence, the projected deceleration is considerable, implying that decreases in emissions and death rates calculated 
here are small rather than large.

a)

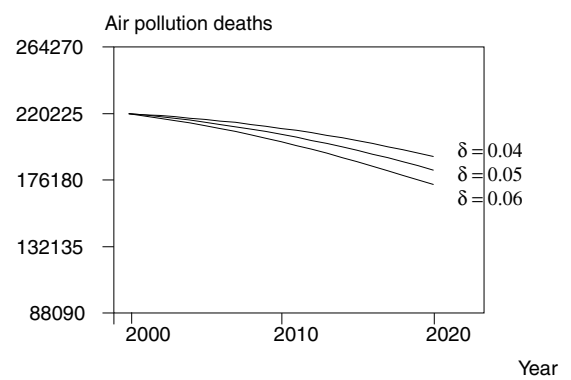

b)

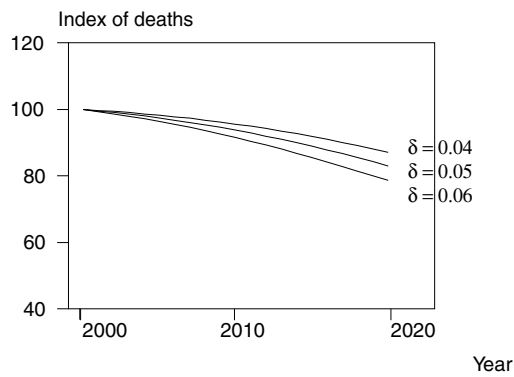

Figure 7: Sensitivity analysis in terms of $\delta$; total number and index of deaths.

All the above results are based on the assumption that $\delta=0.05$, thus being subject to considerable uncertainty. To evaluate the magnitude of this uncertainty, we re-ran all estimates for $\delta=0.04$ and $\delta=0.06$. Figure 7 shows the time paths of the total annual deaths in $E U_{14}$ for the alternative values of $\delta$, both as numbers and as an index, the latter showing that the totals in $E U_{14}$ are 87.13, 83.03, and 78.65 for $\delta=0.04, \delta=0.05$, and $\delta=0.06$ respectively. Hence, deaths decrease as $\delta$ increases, because higher depreciations decrease the calculated capital stock $K$ for 1950-2000 (1970-2000), increasing the estimates for the productivity parameters in (20) which implies higher growth for the period 2000-2020. One can also evaluate the sensitivity of the results in terms of all parameters. We give $\theta$ as an example, showing that the $95 \%$ confidence limits for total deaths in 2020 are $171904 \ldots 194715$ with a mean of 182857 ; i.e., the sensitivity of the results is not very considerable for $\theta$.

To put these results into a more general framework, it is necessary to compare them with other studies. One suitable source is the Regional Air pollution the INformation and Simulation model RAINS (currently called GAINS after amending it for greenhouse cases), which is a large-scale simulation model constructed to validate the emission data from the EU Member States. Depending on the scenario details, GAINS gives several projections 


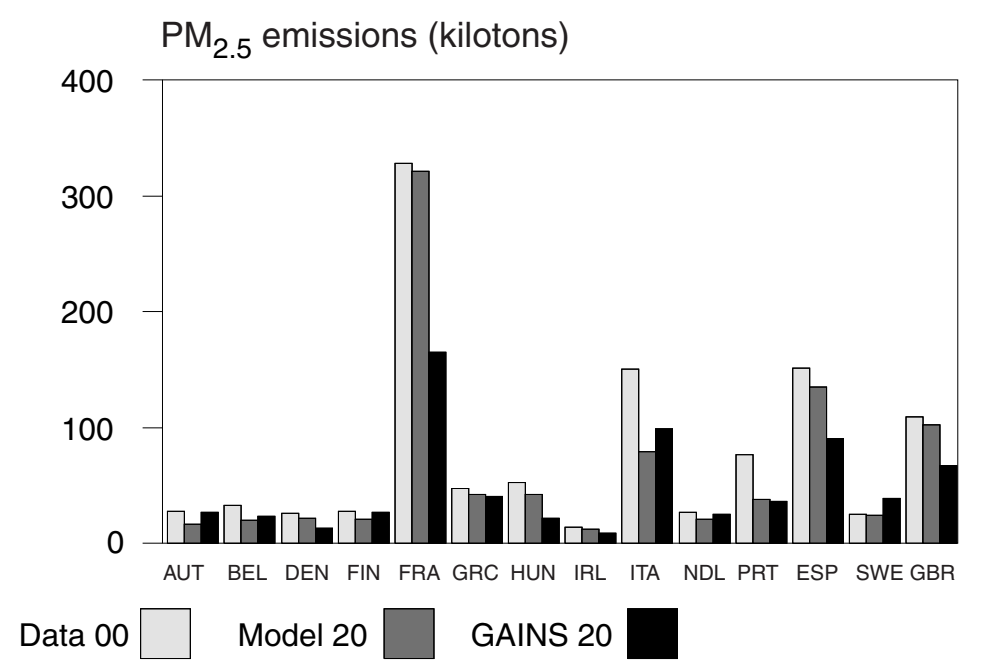

Figure 8: Comparison of emissions.

for $P M_{2.5}$, among which Figure 8, which also shows the data from 2000, compares the Current Legislation scenario with the results calculated by us for 2020. The total emissions in $E U_{14}$ in 2020 in our model (899.65 kilotons) will be larger than in GAINS (686.83 kilotons), this difference having its source in three countries, France (FRA), Spain (ESP) and The United Kingdom (GBR), whereas the other countries show almost similar numbers. Thus one of the future challenges is to pay special attention to those countries where the difference between these two approaches is most significant.

\section{Conclusions}

There is emerging evidence that environmental degradation increases human mortality and a common fear is that economic growth increases the number of environmental deaths by increasing degradation. To evaluate whether this fear is justified, we provide a long-run consumer optimization model in which mortality is endogenous to emissions, which are assumed to follow the $E K C$ path, first rising but then falling along with the output.

The parameters of the model are estimated to accommodate European 
economic data and outdoor air pollution deaths, showing that countries in Europe have reached the downward sloping segment of $E K C$. Economic growth will, therefore, decrease air pollution and the associated death rates in most of the fourteen countries in Europe for which complete analysis is possible. In some countries, however, population growth is so high that the number of deaths will increase. Nevertheless, in spite of the generally decreasing trends, the total number of air pollution deaths from 2000 to 2020 accounts to more than four million in these countries.

Several improvements to the current model are possible, but its simplicity is also an advantage. The simplicity of the production function, for example, reminds us why environmental deaths have been discussed so little: if no considerable productive feedbacks arise, as may be the case in air pollution deaths, then these deaths are human and welfare problems alone. The utilitarian simplicity of the Benthamian function then puts things very bluntly: only the total utility matters and a situation in which some people suffer and die but others go on happily consuming ever more may well be optimal. Policy measures, such as emission limits and international agreements, are thus needed to decrease emissions faster than this study implies, and to prevent the total number of environmental deaths from growing to the kinds of numbers calculated in this paper. 


\section{References}

Amann M, Cofala J, Gzella A, Heyes Ch, Klimont Zb, Schopp W (2007): Estimating concentrations of fine particulate matter in urban background air of European cities. IIASA Interim Report IR-007-01.

Arrow K, Bolin B, Costanza R, Dasgupta P, Folke K, Holling CS, Jansson BO, Levin S, Mäler KG, Perrings C, Pimentel D (1995): Economic Growth, Carrying Capacity, and the Environment. Ecological Economics 15, 91-95.

Aseev SM, Kryazhimskiy AV (2007): The Pontryagin maximum principle and optimal economic growth problems. Moscow: Nauka Publ. MAIK Nauka, Interperiodika.

Brunekreef B, Holgate ST (2002): Air pollution and health. Lancet 360(9341), 1233-1242.

Caselli, F (2004): Accounting for cross-country income differences. CEPR Discussion Paper 4703.

Chay KY, Kenneth Y, Dopkin C, Greenstone M (2003): The Clean Air Act of 1970 and Adult Mortality. Journal of Risk and Uncertainty 27(3), 279-300.

Chay KY, Greenstone M (2003): The impact of air pollution on infant mortality: evidence from geographic variation in pollution shocks induced by a recession. Quarterly Journal of Economics 118, 1121-1167.

Cohen AJ, Anderson RH, Ortro B, Dev Pandey K,Krzyzanowski M, Künzli N, Gutschmidt K, Pope III AC, Romieu I, Samet JM, Smith KR (2004): Mortality Impacts of Urban Air Pollution. In Ezzati M, Lopez AD, Rogers A, Murray CLJ (eds). Comparative Quantification of Health Risks: Global and Regional Burden of Disease Attributable to Selected Major Risk Factors. Geneva, WHO vol 2, 1353-1433.

Cole MA, Elliot RJR (2003): Determining the Trade-Composition Effect: The Role of Capital, Labor, and Environmental Regulations. Journal of Environmental Economics and Management 46, 363-383. 
Currie J, Neidell M (2005): Air pollution and infant health: what can we learn from California's recent experience? Quarterly Journal of Economics 120, 1003-1030.

Hall RE (1988): Intertemporal substitution in consumption. Journal of Political Economy 96, 339-357.

Heston A, Summers R, and Aten B (2006): Penn World Table Version 6.2, Center for International Comparison of Production, Income and Prices at the University of Pennsylvania.

Logan WPD (1953): Mortality in London fog incident, 1952. Lancet, 336338.

Mulligan Casey B, Sala-i-Martin X (1991: A Note on the Time-Elimination Method for Solving Recursive Dynamic Economic Models. National Bureau for Economic Research, Working Paper 116.

Grossman GM, Krueger AB (1995): Economic Growth and the Environment. Quarterly Journal of Economics 110, 9353-9377.

Nemery B, Hoet PHM, Nemmar A (2001): The Meuse Valley fog 1930: an air pollution disaster. Lancet 357, 704-708.

Ostro B (2004): Outdoor air pollution: Assessing the Environmental Burden of Disease at National and Local Levels. WHO Environmental Burden of Disease Series, 5 .

Pope CA, Burnett RT, Thun MJ, Calle EE, Krewski D, Ito K, Thurston GD (2002): Lung cancer, cardiopulmonary mortality, and long-term exposure to fine particulate air pollution. Journal of the American Medical Association $287(9), 1132-1141$.

Samet JM, Dominici F, Curriero FC, Coursac I, Zeger SL (2000): Fine particulate air pollution and mortality in 20 U.S. cities, 1987-1994. The New England Journal of Medicine 343, 1742-1749. 
Selden TM, Song D (1994): Environmental Quality and Development: Is There a Kuznets Curve for Air Pollution Emissions? Journal of Environmental Economics and Management 27, 147-162.

Stokey NL (1998): Are There Limits to Growth? International Economic Review 39, 1-31.

Uzawa H (1968): Time preference, the consumption function, and optimum asset holdings. In J. Wolfe (Editor), Value, capital, and growth. Aldine, Chicago (Chapter 21).

WHO (2004): Meta-analysis of time-series studies and panel studies of particulate matter (PM) and Ozone (O3). World Health Organization, Regional Office for Europe, Copenhagen.

World Bank (2008): World Development Indicators. http://www.worldbank.org. 


\section{A Appendix: Local Stability of the Steady State}

Consider the system of equations (6) and (15). To simplify the notations, we write $\dot{K}=\varphi(K, C)$ and $\dot{C} / C=\psi(K, C)$. The Jacobian of the system is then

$$
J=\left[\begin{array}{ll}
\varphi_{K} & \varphi_{C} \\
\psi_{K} & \psi_{C}
\end{array}\right]
$$

As evaluated around the steady state, its elements become

$$
\begin{aligned}
\varphi_{K}= & \alpha A K^{\alpha-1}-\delta \\
\varphi_{C}= & -1, \\
\psi_{K}= & \frac{1}{\theta}\left\{\frac{d\left[\theta n^{\prime} g^{\prime} \alpha A K^{\alpha-1} /(\rho-\theta n)\right]}{d K}\left(\frac{\theta C}{1-\theta}+A K^{\alpha}-\delta K\right)\right. \\
& \left.+\frac{\theta n^{\prime} g^{\prime} \alpha A K^{\alpha-1}}{\rho-\theta n}\left(\alpha A K^{\alpha-1}-\delta\right)+\alpha(\alpha-1) A K^{\alpha-2}-\theta n^{\prime}\right\}, \\
\psi_{C}= & \frac{1}{1-\theta} \frac{\theta n^{\prime} g^{\prime} \alpha A K^{\alpha-1}}{\rho-\theta n} .
\end{aligned}
$$

Because $\psi_{K}$ necessarily contains the unspecified second derivative $n^{\prime \prime}$, we write

$$
\begin{aligned}
\operatorname{DET} J & =\varphi_{K} \cdot \psi_{C}-\psi_{K} \cdot \varphi_{C} \\
& =\left[\left(-\frac{\varphi_{K}}{\varphi_{C}}\right)-\left(-\frac{\psi_{K}}{\psi_{C}}\right)\right]\left(-\varphi_{C}\right) \cdot \psi_{C} .
\end{aligned}
$$

Consider the steady states depicted in Figure 1. In the single steady state shown in panel $a$, the expression $\left(-\varphi_{C}\right) \cdot \psi_{C}=\frac{1}{1-\theta} \frac{\theta n^{\prime} g^{\prime} \alpha A K^{\alpha-1}}{\rho-\theta n}$ is positive. The square brackets give the difference in the slopes of $\dot{K}=0$ and $\dot{C} / C=0$. Since $\dot{C} / C=0$ hits $\dot{K}=0$ from above, this is positive, implying that $D E T J<0$. Hence, the single steady state is a saddle. By analogous reasoning, steady state 3 in panel $\mathrm{b}$ is also a saddle. In steady state 1 shown in panel b, $\dot{C} / C=0$ hits $\dot{K}=0$ from below, making the expression in the square brackets negative. But since $\frac{1}{1-\theta} \frac{\theta n^{\prime} g^{\prime} \alpha A K^{\alpha-1}}{\rho-\theta n}>0$, this steady state is again a saddle. 
In steady state 2 in panel b, $\dot{C}=0$ hits $\dot{K}=0$ from above (square brackets positive) and $\frac{1}{1-\theta} \frac{\theta n^{\prime} g^{\prime} \alpha A K^{\alpha-1}}{\rho-\theta n}>0$ so that $D E T J>0$. Since all steady states are allocated on the upward sloping segment of $\dot{K}=0$ with $\alpha A K^{\alpha-1}-\delta>0$, and since $g^{\prime}>0$ for steady state 2 , the trace of $J$ given by

$$
\begin{aligned}
T R J & =\varphi_{K}+\psi_{C} \\
& =\alpha A K^{\alpha-1}-\delta+\frac{1}{1-\theta} \frac{\theta n^{\prime} g^{\prime} \alpha A K^{\alpha-1}}{\rho-\theta n}
\end{aligned}
$$

is positive, implying that steady state 2 is unstable. Since the sign of $(T R J)^{2}-4 \cdot D E T J$ is unknown, steady state 2 can be either a focus or a node.

The dynamics outside the steady state are: because $\varphi_{C}=-1$, the capital stock increases (decreases) below (above) phase line $\dot{K}=0$. The behavior of consumption is given by $\psi_{C}=\frac{1}{1-\theta} \frac{\theta n^{\prime} g^{\prime} \alpha A K^{\alpha-1}}{\rho-\theta n}$. Consumption thus increases (decreases) above (below) the phase line $\dot{C} / C=0$ for $K<\mu$, whereas for $K>\mu$ this behavior is reversed. Hence, the stable saddle path which starts from the origin approaches the saddle-stable steady state from the south-west whereas the other approaches it from the north-east. 


\section{B Appendix: Countries and Variables}

\begin{tabular}{l|r|rrr}
\hline \hline Country & Isocode & $P M_{2.5}$ & Death rate & Deaths \\
\hline Austria & AUT & 28.18 & 0.00068 & 5508 \\
Belgium & BEL & 32.86 & 0.00125 & 12904 \\
Denmark & DNK & 25.97 & 0.00061 & 3274 \\
Finland & FIN & 28.26 & 0.00025 & 1272 \\
France & FRA & 328.23 & 0.00071 & 42202 \\
Greece & GRC & 47.32 & 0.00066 & 7242 \\
Hungary & HUN & 52.38 & 0.00126 & 12895 \\
Ireland & IRL & 14.16 & 0.00031 & 1174 \\
Italy & ITA & 150.27 & 0.00088 & 50766 \\
Netherlands & NLD & 26.78 & 0.00098 & 15573 \\
Portugal & PRT & 76.99 & 0.00049 & 5053 \\
Spain & ESP & 151.14 & 0.00049 & 19976 \\
Sweden & SWE & 25.40 & 0.00037 & 3284 \\
United Kingdom & GBR & 109.40 & 0.00068 & 39543 \\
\hline EU $U_{14}$ (Total / Average) & & 1097.34 & 0.00069 & 220225 \\
\hline Cyprus & CYP & 2.18 & 0.00030 & 231 \\
Czech Rep. & CZE & 42.69 & 0.00088 & 9086 \\
Estonia & EST & 21.69 & 0.00044 & 631 \\
Germany & GER & 159.86 & 0.00091 & 75150 \\
Latvia & LVA & 10.93 & 0.00055 & 1334 \\
Lithuania & LTU & 12.50 & 0.00061 & 2197 \\
Luxembourg & LUX & 2.73 & 0.00074 & 321 \\
Malta & MLT & 0.59 & 0.00049 & 193 \\
Poland & POL & 202.70 & 0.00085 & 32944 \\
Slovak Rep. & SVK & 14.50 & 0.00079 & 4265 \\
Slovenia & SVN & 12.08 & 0.00082 & 1582 \\
\hline EU $U_{25}$ (Total / Average) & & 1579.79 & 0.00068 & 348600 \\
\hline \hline
\end{tabular}

All numbers refer to year 2000. $P M_{2.5}$ emissions in kilotons (Amann et al. 2007), Deaths refer to air pollution induced deaths (WHO 2004). In addition, annual series from 1950 (from 1970 for Hungary) for population, GDPpc, and investments (Heston et al. 2006). 